\title{
A SPECTRAL SEQUENCE FOR THE HOMOTOPY OF NICE SPACES $\left(^{1}\right)$
}

\author{
BY

\section{A. K. BOUSFIELD AND E. B. CURTIS}

1. Introduction. Using the approach of $[\mathrm{C}],[\mathrm{R}]$ and $[6 \mathrm{~A}]$, we construct for each space $X$, a spectral sequence converging (almost always) to the homotopy groups of $X$ modulo odd torsion. This can be considered an "unstable Adams spectral sequence" in that it agrees with the ordinary mod-2 Adams spectral sequence $[A]$ for a stable range of dimensions. Furthermore our spectral sequence agrees with that of [M,P] when theirs is defined. However our version has these advantages: it is more generally defined, has an $E_{1}$-term suitable for computation, and has an $E_{2}$-term which is homologically describable for nice spaces (see 2.2).

Our statements and constructions are in terms of simplicial sets, simplicial groups etc. Corresponding statements for topological spaces may be obtained by means of the singular complex and geometric realization functors.

For each connected simplicial set $K$, the spectral sequence $\left[E_{r} K\right]$ arises from the homotopy exact couple associated with a modified lower central series filtration of Kan's simplicial loop group $G K$. If $K$ is simply connected with finitely generated homotopy groups, then $\left\{E_{r} K\right\}$ converges to $\pi_{*} K$ modulo odd torsion. In order to describe $E_{2} K$ homologically, we assume that $H_{*}\left(K ; Z_{2}\right)$ is a nice homology coalgebra as in [B]. This condition is satisfied, for example, when $H^{*}\left(K ; Z_{2}\right)$ factors as the tensor product of polynomial and truncated polynomial algebras. Under the niceness assumption, we construct a fundamental chain complex whose homology is $E_{2} K$. This complex, and hence $E_{2} K$, depends only on $H_{*}\left(K ; Z_{2}\right)$ as a coalgebra over the Steenrod algebra.

The paper is in two parts. In Part I, we construct the spectral sequence and the fundamental chain complex. We derive a simplified $E_{1}$-term in the Massey-Peterson case, and then give our homological interpretation of $E_{2} K$ when $H_{*}\left(K ; Z_{2}\right)$ is nice. In Part II, we prove that the fundamental chain complex serves as an $E_{1}$-term for our spectral sequence. The proof involves a detailed study of a closely related spectral sequence defined by Rector [R].

Using a very different approach from ours, D. M. Kan and the first author $[\mathrm{B}, \mathrm{K}]$ have developed a mod- $p$ spectral sequence, for all primes $p$, which has the

Received by the editors June 15, 1969.

(1) Research supported in part by NSF GP 8885 , NSF GP 9565 , and by J. S. Guggenheim Fellowship. 
advantage that, without any niceness assumption, the $E_{2}$-term admits a homological description (as in §6). On the other hand, the advantage of our present work is that for nice spaces the $E_{2}$-term is more accessible, as the homology of the fundamental chain complex. This becomes useful in the examples (forthcoming) of $S^{n}, S U(n), B U(n), S O(n), B O(n)$, Stiefel manifolds, and especially $S F, B S F$.

The authors are grateful to D. M. Kan for his advice and criticisms.

1.1. Conventions. Let $K$ be a reduced simplicial set; that is, $K$ has only one vertex. Let $H_{*} K$ denote the homology of $K$ with $Z_{2}$ coefficients. The diagonal map $K \rightarrow K \times K$ induces a comultiplication

$$
\Delta: H_{*} K \rightarrow H_{*} K \otimes H_{*} K
$$

making $H_{*} K$ a homology coalgebra, that is, a connected cocommutative coalgebra. Also $H_{*} K$ is a right module over the Steenrod algebra, $A$, with operations

$$
\text { (·) } S q^{i}: H_{n} K \rightarrow H_{n-i} K
$$

which dualize to the usual Steenrod operations in cohomology. Similar conventions apply to the reduced homology $\tilde{H}_{*} K$.

PART I. A SPECTRAL SEQUENCE FOR THE hOMOTOPY OF NICE SPACES.

2. The spectral sequence and the fundamental chain complex. To construct the spectral sequence, we first recall that the mod-2 lower central series of a group $G$ is the filtration (see $[R]$ )

$$
G=\Gamma_{1} G \supset \cdots \supset \Gamma_{s} G \supset \Gamma_{s+1} G \supset \ldots
$$

where $\Gamma_{s} G$ is the subgroup of $G$ generated by all $\left\langle g_{1}, \ldots, g_{k}\right\rangle^{2^{t}}$ such that $2^{t} k \geqq s$, the $g_{i} \in G$, and $\langle, \ldots$,$\rangle is the simple commutator. For a reduced simplicial set$ $K$, the loop group $G K$ (defined in $[\mathrm{K}]$ ) is a free simplicial group for which

$$
\pi_{q-1}(G K) \approx \pi_{q}(K), \quad \pi_{q-1}\left(G K / \Gamma_{2} G K\right) \approx \tilde{H}_{q}(K)
$$

We filter $G K$ (in each dimension) by the modified mod-2 lower central series

$$
G K \supset \Gamma_{2} G K \supset \ldots \supset \Gamma_{2^{s}} G K \supset \Gamma_{2^{s+1}} G K \supset \cdots .
$$

The associated homotopy exact couple gives rise to a spectral sequence $\left\{E_{\mathrm{r}} K\right\}$ :

$$
E_{1}^{s, t}(K)=\pi_{t-s-1}\left(\Gamma_{2^{s}} G K / \Gamma_{2^{s+1}} G K\right), \quad d_{r}: E_{r}^{s, t}(K) \rightarrow E_{r}^{s+r, t+r-1}(K) .
$$

Let $\pi_{*}(K ; 2)$ denote the quotient of $\pi_{*} K$ by the subgroup of elements of odd finite order. The following theorem summarizes results of $[R]$ and $[6 \mathrm{~A}]$.

THEOREM 2.1. If $K$ is simply connected with finitely generated homotopy groups, then $\left\{E_{r} K\right\}$ converges to $\pi_{*}(K ; 2)$. More precisely $E_{r}^{s, t}(K)=E_{\infty}^{s, t}(K)$ for large $r$, and $\left\{E_{\infty}^{s, s+n}(K)\right\}_{s \geq 0}$ is the graded group of a complete decreasing filtration of $\pi_{n}(K ; 2)$. For $r \geqq 2,\left\{E_{r} K\right\}$ coincides in the stable range with the mod-2 Adams spectral sequence.

2.2. Nice homology coalgebras. In general $E_{2} K$ seems to depend on the "higher order" structure of $H_{*} K$; but this difficulty is avoided when $H_{*} K$ is a nice homology 
coalgebra, as in [B]. To recall the notion of niceness, we first suppose that $C$ is a homology coalgebra of finite type over $Z_{2}$; that is, $C$ is finitely generated in each dimension. Then $C$ is nice if and only if the dual algebra $C^{*}$ has a presentation $C^{*} \approx R / I$ where $R$ is a connected polynomial algebra of finite type over $Z_{2}$ and $I$ is a Borel ideal (see [S, p. 79]). By [S, p. 80] this is equivalent to the condition that $C^{*} \approx R \otimes_{B} Z_{2}$ where $R$ is as above, $B$ is a polynomial subalgebra of $R$, and $R$ is free as a $B$-module. The definition of niceness for an arbitrary homology coalgebra over $Z_{2}$ is obtained by suitably dualizing the foregoing condition on $C^{*}$. We remark that by [B, 4.1], a homology coalgebra $C$ is nice if and only if $R^{n} P(C)=0$ for $n>1$ where $R^{n} P$ is the $n$th right derived functor of the primitive element functor.

Some (overlapping) examples of nice coalgebras are given by:

(i) If $H_{*} K$ is the coalgebra of some Hopf algebra, then $H_{*} K$ is nice.

(ii) If $H^{*} K$ is of finite type and factors as a tensor product of polynomial and truncated polynomial algebras, then $H_{*} K$ is nice.

In particular if $K$ is homotopy equivalent to a loop space or to a sphere, then $H_{*} K$ is nice. We sometimes call a space nice if its homology is nice.

2.3. The algebra $\Lambda$. Our fundamental chain complex for "computing" $E_{2} K$ when $K$ nice will involve the algebra $\Lambda\left(=E^{1}(S)\right.$ in [6A]). Recall that $\Lambda$ is the graded associative differential algebra with unit (over $Z_{2}$ ) with:

(i) a generator $\lambda_{i}$ of degree $i$ for each $i \geqq 0$,

(ii) for each $i, k \geqq 0$ a relation

$$
\lambda_{1} \lambda_{2 i+1+k}=\sum_{j \geq 0}\left(\begin{array}{c}
k-1-j \\
j
\end{array}\right) \lambda_{i+k-j} \lambda_{2 i+1+j},
$$

(iii) a differential $\partial$ given by

$$
\partial\left(\lambda_{i}\right)=\sum_{j \geqq 1}\left(\begin{array}{c}
i-j \\
j
\end{array}\right) \lambda_{i-j} \lambda_{j-1} .
$$

Let $I=\left(i_{1}, \ldots, i_{s}\right)$ be a finite sequence of nonnegative integers. Call $I$ allowable if $i_{j+1} \leqq 2 i_{j}$ for each $0<j<s$, or if $I$ is empty. Then $\Lambda$ has a $Z_{2}$-basis given by all $\lambda_{I}=\lambda_{i_{1}} \cdots \lambda_{i_{s}}$ with allowable $I\left(\lambda_{I}=1\right.$ when $I$ empty). Note that $\Lambda=\bigoplus_{s \geq 0} \Lambda^{s}$ where $\Lambda^{s}$ is generated by the monomials $\lambda_{I}$ of length $s$. For $n \geqq 0$ let $\Lambda(n)$ be the subspace of $\Lambda$ generated by all allowable $\lambda_{I}$ for which $i_{1}<n$ or $I$ empty. Each $\Lambda(n)$ is closed under $\partial$, and in fact $\Lambda(n) \approx E_{1}\left(S^{n}\right)$ by [6A].

Notation. For a graded $Z_{2}$-module $M$, let $M \tilde{\otimes} \Lambda$ (resp. $M \hat{\otimes} \Lambda$ ) be the subspace of $M \otimes \Lambda$ generated by all $x \otimes \alpha$ with $x \in M_{n}$ and $\alpha \in \Lambda(n-1)$ (resp. $\alpha \in \Lambda(n)$ ). Also let $M \tilde{\otimes} \Lambda^{s}$ and $M \hat{\otimes} \Lambda^{s}$ be the obvious summands.

2.4. The functor $\Psi$. A certain nonadditive functor $\Psi$ will appear in our fundamental chain complex (2.5). The map

$$
\Delta \otimes 1+1 \otimes \Delta: \tilde{H}_{*} K \otimes \tilde{H}_{*} K \rightarrow \tilde{H}_{*} K \otimes \tilde{H}_{*} K \otimes \tilde{H}_{*} K
$$

restricts to a homomorphism $\delta^{\Delta}: L_{2}\left(\tilde{H}_{*} K\right) \rightarrow L_{3}\left(\tilde{H}_{*} K\right)$ where

$$
L(\cdot)=\bigoplus_{r \geqq 1} L_{r}(\cdot)
$$


is the free restricted Lie algebra functor as in 7.2. We define

$$
\Psi\left(H_{*} K\right)=\operatorname{Ker} \delta^{\Delta} .
$$

The right action of the Steenrod algebra $A$ on $H_{*}(K \times K) \approx H_{*} K \otimes H_{*} K$ induces a right $A$-module structure on $\Psi\left(H_{*} K\right)$, and the diagonal $\Delta$ restricts to a right $A$-module map

$$
\Delta: \tilde{H}_{*} K \rightarrow \Psi\left(H_{*} K\right) .
$$

Let $\sigma: \Psi\left(H_{*} K\right) \rightarrow \tilde{H}_{*} K$ be the restriction of the homomorphism (halving degrees) $L_{2}\left(\tilde{H}_{*} K\right) \rightarrow \tilde{H}_{*} K$ which sends $x^{[2]} \rightarrow x$ and $[x, y] \rightarrow 0$ for $x, y \in \tilde{H}_{*} K$.

2.5. The fundamental chain complex. The following chain complex $W\left(H_{*} K\right)$ will serve as an $E_{1}$-term for our spectral sequence when $H_{*} K$ is nice. Define for each $s \geqq 0$

$$
\begin{gathered}
W^{s}\left(H_{*} K\right)=\left(\tilde{H}_{*} K \tilde{\otimes} \Lambda^{s}\right) \oplus\left(\Psi\left(H_{*} K\right) \tilde{\otimes} \Lambda^{s-1}\right), \\
\delta: W^{s}\left(H_{*} K\right) \rightarrow W^{s+1}\left(H_{*} K\right),
\end{gathered}
$$

where the differential $\delta$ has the following components:

(i) for $x \otimes \lambda_{I} \in H_{n} K \tilde{\otimes} \Lambda^{s}$,

$$
\delta\left(x \otimes \lambda_{I}\right)=\left(x \otimes \partial \lambda_{I}+\sum_{i=1}^{m} x S q^{i} \otimes \lambda_{i-1} \lambda_{I}\right) \oplus\left(\Delta x \otimes \lambda_{I}\right)
$$

where $m=[(n-1) / 2]$;

(ii) for $x \otimes \lambda_{I} \in \Psi\left(H_{*} K\right) \tilde{\otimes} \Lambda^{s-1}$ when $\operatorname{deg} x=2 m+1$

$$
\delta\left(x \otimes \lambda_{I}\right)=(0) \oplus\left(x \otimes \partial \lambda_{I}+\sum_{i \geqq 1} x S q^{i} \otimes \lambda_{i-1} \lambda_{I}\right)
$$

and when $\operatorname{deg} x=2 m+2$

$$
\begin{aligned}
\delta\left(x \otimes \lambda_{I}\right)= & \left(\sigma x \otimes\left(\partial \lambda_{m}\right) \lambda_{I}+\sum_{i \geqq 1}(\sigma x) S q^{i} \otimes\left(\lambda_{i-1} \lambda_{m}+\lambda_{m-i} \lambda_{2 i-1}\right) \lambda_{I}\right) \\
& \oplus\left(x \otimes \partial \lambda_{I}+\sum_{i=1}^{m} x S q^{i} \otimes \lambda_{i-1} \lambda_{I}\right) .
\end{aligned}
$$

We give $W\left(H_{*} K\right)$ a bigradation such that $x \otimes \lambda_{i_{1}} \cdots \lambda_{i_{s}}$ is of bidegree $(s, t)$ with

$$
t=s+\operatorname{deg} x+\sum_{j=1}^{s} i_{j}
$$

Our main result is the following.

THEOREM 2.6. Let $K$ be a reduced simplicial set such that $H_{*} K$ is a nice coalgebra. Then there are natural isomorphisms

$$
E_{2}^{s, t}(K) \approx H^{s, t}\left(W\left(H_{*} K\right)\right)
$$

for all $s, t$.

This will be proved in $\S 13$. 
COROLlary 2.7. If $H_{*} K$ is nice then $E_{2} K$ depends only on the structure of $H_{*} K$ as a coalgebra over the Steenrod algebra.

3. Unstable modules over the Steenrod algebra. The problem of computing $E_{2} K$ via the fundamental chain complex will often reduce (see $\left.\$ \$ 4-5\right)$ to that of computing Ext groups for unstable modules over the Steenrod algebra $A$.

Definition 3.1. An unstable right $A$-module consists of a graded right $A$-module $M\left(M_{n}=0\right.$ for $\left.n<0\right)$ such that $x S q^{i} \in M_{n-i}$ for $x \in M_{n}$ and $x S q^{i}=0$ for $2 i>n$.

Let $\mathscr{M} A$ denote the category of unstable right $A$-modules, and note that each $\tilde{H}_{*} K \in \mathscr{M} A$. It is straightforward to show that $\mathscr{M} A$ is an abelian category with enough injectives. The sth right derived functor of

$$
\operatorname{Hom}_{\mathscr{M}_{A}}\left(\tilde{H}_{*}\left(S^{t}\right), \cdot\right)
$$

will be called

$$
\operatorname{Ext}_{\mathscr{M}_{A}}^{s, t}\left(Z_{2}, M\right)
$$

for $s, t \geqq 0$ and $M \in \mathscr{M} A$.

3.2. A complex for Ext. A simplified form of the fundamental chain complex may be used to compute the above Ext. For $M \in \mathscr{M} A$, let $V(M)$ be the chain complex with

$$
\begin{aligned}
V^{s}(M) & =M \hat{\otimes} \Lambda^{s}, \quad s \geqq 0, \\
\delta\left(x \otimes \lambda_{I}\right) & =x \otimes \partial \lambda_{I}+\sum_{i \geqq 1} x S q^{i} \otimes \lambda_{i-1} \lambda_{I} .
\end{aligned}
$$

As before $x \otimes \lambda_{i_{1}} \cdots \lambda_{i_{s}}$ is given bidegree $(s, t)$ with $t=s+\operatorname{deg} x+\sum_{j=1}^{s} i_{j}$.

THEOREM 3.3. For $M \in \mathscr{M} A$ and $s, t \geqq 0$ there is a natural isomorphism

$$
\operatorname{Ext}_{\mathscr{M}_{A}}^{s, t}\left(Z_{2}, M\right) \approx H^{s, t}(V(M)) .
$$

This will be proved in 3.6.

3.4. The functors $S, \Omega$ and $\Omega^{1}$. The following definitions are dual to those of [M,P]. For $M \in \mathscr{M} A$ let $S M \in \mathscr{M} A$ be given by $(S M)_{i}=M_{i-1}$ with the same $A$ action as $M$. This "suspension" functor has a right adjoint "loop" functor $\Omega$. Define $\Omega M \in \mathscr{M} A$ by

$$
(\Omega M)_{2 i}=M_{2 i+1}, \quad(\Omega M)_{2 i-1}=\operatorname{Ker}\left(S q^{i}: M_{2 i} \rightarrow M_{i}\right)
$$

with $A$ action on $\Omega M$ induced by that on $M$. The functor $\Omega$ is left exact and has a first derived functor $\Omega^{1}$, where $\Omega^{1} M \in \mathscr{M} A$ is defined by

$$
\left(\Omega^{1} M\right)_{2 i}=0, \quad\left(\Omega^{1} M\right)_{2 i-1}=\operatorname{Coker}\left(S q^{i}: M_{2 i} \rightarrow M_{i}\right)
$$

with

$$
S q^{2 j}:\left(\Omega^{1} M\right)_{2 i-1} \rightarrow\left(\Omega^{1} M\right)_{2 i-2 j-1}
$$

induced by $S q^{j}: M_{i} \rightarrow M_{i-j}$. 
There is a natural inclusion $i: \Omega M \rightarrow M$ raising degree by one, and a natural projection $p: M \rightarrow \Omega^{1} M$ sending degree $d$ to degree $2 d-1$.

Note that if

$$
0 \rightarrow M^{\prime} \rightarrow M \rightarrow M^{\prime \prime} \rightarrow 0
$$

is a short exact sequence in $M A$, there is induced an exact sequence in $\mathscr{M} A$

$$
0 \rightarrow \Omega M^{\prime} \rightarrow \Omega M \rightarrow \Omega M^{\prime \prime} \rightarrow \Omega^{1} M^{\prime} \rightarrow \Omega^{1} M \rightarrow \Omega^{1} M^{\prime \prime} \rightarrow 0 .
$$

The following lemma, together with 3.3, will often provide an inductive procedure for computing $\operatorname{Ext}_{\mathcal{M A}_{A}}\left(Z_{2}, M\right)$.

Lemma 3.5. Let $M \in \mathscr{M} A$ with $M_{0}=0$. Then there is a natural exact sequence

$$
\begin{aligned}
\cdots \rightarrow H^{s, t}(V(\Omega M)) & \rightarrow H^{s, t+1}(V(M)) \rightarrow H^{s-1, t}\left(V\left(\Omega^{1} M\right)\right) \\
& \rightarrow H^{s+1, t}(V(\Omega M)) \rightarrow H^{s+1, t+1}(V(M)) \rightarrow \cdots .
\end{aligned}
$$

Proof. Filter $V(M)$ by subcomplexes $F_{2} V(M) \subset F_{1} V(M) \subset V(M)$ as follows:

$$
\begin{aligned}
& F_{2} V(M)=\operatorname{Image}(V(i): V(\Omega M) \rightarrow V(M)), \\
& F_{1} V^{s}(M)=\operatorname{Ker}\left(h^{s}: V^{s}(M) \rightarrow V^{s-1}\left(\Omega^{1} M\right)\right),
\end{aligned}
$$

where for $x \in M_{n+1}, \lambda_{I}=\lambda_{i_{1}} \cdots \lambda_{i_{s}}$,

$$
\begin{aligned}
h^{s}\left(x \otimes \lambda_{I}\right) & =0, & & i_{1}<n, \\
& =p(x) \otimes \lambda_{i_{2}} \cdots \lambda_{i_{s}}, & & i_{1}=n .
\end{aligned}
$$

It is straightforward to verify that the chain complex $F_{1} V(M) / F_{2} V(M)$ is acyclic. The lemma now follows from the isomorphisms

$$
\begin{aligned}
H^{s}\left(F_{2} V(M)\right) & \approx H^{s}(V(\Omega M)), \\
H^{s}\left(V(M) / F_{1} V(M)\right) & \approx H^{s-1}\left(V\left(\Omega^{1} M\right)\right) .
\end{aligned}
$$

3.6. Proof of 3.3. A short exact sequence

$$
0 \rightarrow M^{\prime} \rightarrow M \rightarrow M^{\prime \prime} \rightarrow 0
$$

in $\mathscr{M} A$ gives a natural exact sequence

$$
\cdots \rightarrow H^{s, t}\left(V\left(M^{\prime}\right)\right) \rightarrow H^{s, t}(V(M)) \rightarrow H^{s, t}\left(V\left(M^{\prime \prime}\right)\right) \rightarrow H^{s+1, t}\left(V\left(M^{\prime}\right)\right) \rightarrow \cdots .
$$

Thus it suffices to show when $M$ is injective in $\mathscr{M} A$ that

$$
\begin{aligned}
& H^{s, t}(V(M))=0 \text { for } s>0, \\
& H^{0, t}(V(M)) \approx \operatorname{Hom}_{\mathscr{K}_{A}}\left(\tilde{H}_{*}\left(S^{t}\right), M\right) .
\end{aligned}
$$

The second isomorphism is evident. For the first, for each $n \geqq 0$, let $I(n)$ be the injective unstable $A$-module on one $n$-dimensional generator $(I(n)$ is dual to $F(n)$ in [S,E, p. 27]). For $n \geqq 1, \Omega I(n)=I(n-1)$ and $\Omega^{1} I(n)=0$. Hence 3.5 applies to 
show inductively that $H^{s, t}(V I(n))=0$ for $s>0, n \geqq 0$. Since any injective $M \in \mathscr{M} A$ is a retract of a direct sum $\oplus_{\alpha} I\left(n_{\alpha}\right)$, it follows that $H^{s, t}(V(M))=0$ for $s>0$.

4. A generalized $E H P$ sequence. Let $K$ be a reduced simplicial set such that the coalgebra $H_{*} K$ is nice. We shall construct an exact sequence involving $E_{2} K$ and certain Ext terms. When $K$ is a sphere, this will give a kind of $E H P$ sequence on the $E_{2}$ level (see 5.3).

Let $\Delta: \tilde{H}_{*} K \rightarrow \Psi\left(H_{*} K\right)$ be as in 2.4 and define

$$
\bar{P}\left(H_{*} K\right)=\Omega(\operatorname{Ker} \Delta), \quad \bar{R}\left(H_{*} K\right)=\Omega(\text { Coker } \Delta)
$$

where $\Omega$ is as in 3.4. Note that $\bar{P}\left(H_{*} K\right)$ consists of the primitive elements in $H_{*} K$ with gradations lowered by one; and that $\bar{R}\left(H_{*} K\right)_{t}=P_{-2, t+1}\left(H_{*} K\right)$ in the notation of $[\mathrm{M}, \mathrm{S}, \mathrm{II}]$.

THEOREM 4.1. If $H_{*} K$ is nice there is a natural exact sequence

$$
\begin{aligned}
\cdots \rightarrow \operatorname{Ext}_{\mathscr{M}_{A}}^{s, t}\left(Z_{2}, \bar{P} H_{*} K\right) \rightarrow E_{2}^{s, t+1}(K) \rightarrow \operatorname{Ext}_{\mathscr{M}_{A}^{-1, t}}^{s}\left(Z_{2}, \bar{R} H_{*} K\right) \\
\\
\rightarrow \operatorname{Ext}_{\mathscr{M}_{A}}^{s+1, t}\left(Z_{2}, \bar{P} H_{*} K\right) \rightarrow \cdots
\end{aligned}
$$

Proof. Filter the fundamental chain complex $W\left(H_{*} K\right)$ by

$$
F_{2} W\left(H_{*} K\right) \subset F_{1} W\left(H_{*} K\right) \subset W\left(H_{*} K\right)
$$

as follows. Let $F_{2} W\left(H_{*} K\right)$ be the image of the inclusion $V\left(\bar{P} H_{*} K\right) \rightarrow W\left(H_{*} K\right)$ induced by the inclusion $\bar{P} H_{*} K \rightarrow H_{*} K$. Let $F_{1} W^{s}\left(H_{*} K\right)$ be the subspace

$$
\left(\tilde{H}_{*} K \tilde{\otimes} \Lambda^{s}\right) \oplus\left(B \tilde{\otimes} \Lambda^{s-1}\right) \subset W^{s}\left(H_{*} K\right)
$$

where $B$ is the image of $\Delta: \tilde{H}_{*} K \rightarrow \Psi\left(H_{*} K\right)$. It is straightforward to show that

$$
\begin{aligned}
H^{s, t}\left(F_{2} W\left(H_{*} K\right)\right) & \approx H^{s, t-1}\left(V\left(\bar{P} H_{*} K\right)\right), \\
H^{s, t}\left(F_{1} W\left(H_{*} K\right) / F_{2} W\left(H_{*} K\right)\right) & =0, \\
H^{s, t}\left(W\left(H_{*} K\right) / F_{1} W\left(H_{*} K\right)\right) & \approx H^{s-1, t-1}\left(V\left(\bar{R} H_{*} K\right)\right)
\end{aligned}
$$

and the result now follows from 3.3.

COROLlaRY 4.2. If $H^{*} K$ is a polynomial algebra of finite type, then

$$
E_{2}^{s, t}(K) \approx \operatorname{Ext}_{\mathscr{M}_{A}^{s-1}}^{s-1}\left(Z_{2}, \bar{P} H_{*} K\right) .
$$

Proof. This follows from 4.1 since $\bar{R}\left(H_{*} K\right)=0$.

5. On $E_{2} K$ in the Massey-Peterson case. Massey and Peterson in [M,P] constructed their unstable Adams spectral sequence for each space $K$ such that $H^{*} K$ is a free unstable $A$-algebra [S,E, p. 29]. In particular for such $K, H_{*} K$ is nice, and we shall show that our $E_{2} K$ is the same as theirs. Also for such $K, 3.3$ provides a convenient $E_{1}$-term. 
Let $\mathscr{C} A$ be the category of unstable right $A$-module homology coalgebras. That is, each $C \in \mathscr{C} A$ is simultaneously an unstable right $A$-module and a connected cocommutative coalgebra where the structures are compatible as follows. The comultiplication $\Delta$ of $C$ satisfies a Cartan formula; and

$$
\sqrt{ } \cdot=(\cdot) S q^{n}: C_{2 n} \rightarrow C_{n}
$$

where the "square root" map $\sqrt{ }$. is dual to the squaring map for commutative $Z_{2}$-algebras. Of course $H_{*} K \in \mathscr{C} A$ if $K$ is a reduced simplicial set.

5.1. The functor $U_{*}$. For $M \in \mathscr{M} A$ with $M_{0}=0$, define $U_{*}(M) \in \mathscr{C} A$ together with $j: U_{*}(M) \rightarrow M$ by the universal property:

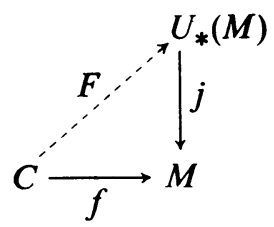

i.e., for any such $f \in \mathscr{M} A$, there exists a unique $F \in \mathscr{C} A$ such that $j \circ F=f$. If $M$ is of finite type, then $U_{*}(M)$ is dual to $U\left(M^{*}\right)$ where $U$ is the free unstable $A$-algebra functor [S,E, p. 29].

THEOREM 5.2. If $K$ is a reduced simplicial set with $H_{*} K \approx U_{*}(M)$ in $\mathscr{C} A$, then

$$
E_{2}^{s, t}(K) \approx \operatorname{Ext}_{\mathscr{M}_{A}}^{s, t}\left(Z_{2}, M\right)
$$

Proof. Using the notation of 2.5 and 3.5, let $v: W\left(H_{*} K\right) \rightarrow V\left(H_{*} K\right)$ be the chain map

$$
v^{s}=f \oplus g:\left(\tilde{H}_{*} K \tilde{\otimes} \Lambda^{s}\right) \oplus\left(\Psi\left(H_{*} K\right) \tilde{\otimes} \Lambda^{s-1}\right) \rightarrow H_{*} K \hat{\otimes} \Lambda^{s}
$$

where $f$ is the inclusion and

$$
\begin{aligned}
g\left(x \otimes \lambda_{I}\right) & =\sigma x \otimes \lambda_{k} \lambda_{I} & & \text { if } \operatorname{deg} x=2 k+2, \\
& =0 & & \text { if } \operatorname{deg} x=2 k+1 .
\end{aligned}
$$

Define a chain map $u: W\left(H_{*} K\right) \rightarrow V(M)$ by $u=V(j) \circ v, j: H_{*} K \approx U_{*}(M) \rightarrow M$. Since

$$
\bar{P} U_{*}(M) \approx \Omega M, \quad \bar{R} U_{*}(M) \approx \Omega^{1} M,
$$

it is straightforward to show that $u$ induces chain isomorphisms

$$
F_{2} W\left(H_{*} K\right) \approx F_{2} V(M), \quad W\left(H_{*} K\right) / F_{1} W\left(H_{*} K\right) \approx V(M) / F_{1} V(M)
$$

using the filtrations of 3.5 and 4.1. Hence

$$
u^{*}: H^{*}\left(W\left(H_{*} K\right)\right) \approx H^{*}(V(M))
$$

and the theorem follows from 2.6 and 3.3. 
REMARK 5.3. When $H_{*} K \approx U_{*}(M)$ it follows that the exact sequence of 4.1 for $K$ is equivalent to the sequence of 3.5 for $M$. If $K=S^{n}$ this sequence becomes

$$
\cdots \rightarrow E_{2}\left(S^{n-1}\right) \rightarrow E_{2}\left(S^{n}\right) \rightarrow E_{2}\left(S^{2 n-1}\right) \rightarrow \cdots
$$

which is an $E H P$ sequence on the $E_{2}$-level (cf. [C3]).

6. A homological interpretation of $E_{2} K$. When $H_{*} K$ is nice $E_{2} K$ will be described as an Ext using the theory of nonadditive derived functors (see [An] and [B, Appendix]). For any $K$ this Ext also gives the $E_{2}$-term of the unstable Adams spectral sequence constructed in $[\mathrm{B}, \mathrm{K}]$, and thus our $E_{2} K$ coincides with theirs when $H_{*} K$ is nice. More details on the required derived functors may be found in $[\mathrm{B}]$ and $[\mathrm{B}, \mathrm{K}]$.

6.1. Derived functors on $\mathscr{C} A$. Let $\mathscr{C} A$ be the category of unstable $A$-coalgebras described in $\S 5$. An object $I \in \mathscr{C} A$ will be called cofree if $I \approx H_{*} N$ where

$$
N=\prod_{n \geqq 1} K\left(\pi_{n}, n\right)
$$

with each $\pi_{n}$ a $Z_{2}$-module. A cosimplicial resolution of $C \in \mathscr{C} A$ consists of a cosimplicial object $\boldsymbol{X}$ over $\mathscr{C} A$ together with an augmentation $e: C \rightarrow X^{0}$, such that:

(i) $X^{n}$ is cofree for $n \geqq 0$,

(ii) the chain complex of graded $Z_{2}$-modules

$$
C \stackrel{e}{\rightarrow} X^{0} \stackrel{\delta}{\rightarrow} X^{1} \stackrel{\delta}{\rightarrow} X^{2} \stackrel{\delta}{\rightarrow} \cdots
$$

is acyclic, where

$$
\delta=\sum(-1)^{i} d^{i}: X^{n-1} \rightarrow X^{n}
$$

and the $d^{i}$ are the coface operators. Such resolutions exist for all $C \in \mathscr{C} A$. If $T: \mathscr{C A} \rightarrow \mathscr{B}$ is any functor to an abelian category $\mathscr{B}$, then $T$ has right derived functors

For $C \in \mathscr{C} A$,

$$
R^{s} T: \mathscr{C} A \rightarrow \mathscr{B}, \quad s \geqq 0 .
$$

$$
R^{s} T(C) \approx H^{s}(T X), \quad s \geqq 0,
$$

where $(X, e)$ is any cosimplicial resolution of $C$ and $T X$ is the cosimplicial object over $B$ with $(T X)^{n}=T\left(X^{n}\right)$.

For $t \geqq 1$ the functor

$$
\operatorname{Hom}_{\mathscr{C} A}\left(H_{*} S^{t}, \cdot\right): \mathscr{C} A \rightarrow\left(Z_{2} \text {-modules }\right)
$$

has right derived functors

$$
\operatorname{Ext}_{\xi A}^{s, t}\left(Z_{2}, \cdot\right)=\operatorname{Ext}_{\mathscr{\&} A}^{s}\left(H_{*} S^{t}, \cdot\right) \text {. }
$$

THEOREM 6.2. If $K$ is a reduced simplicial set with $H_{*} K$ nice, then there are natural isomorphisms

$$
E_{2}^{s, t}(K) \approx \operatorname{Ext}_{\delta_{A}^{s}, t}^{s}\left(Z_{2}, H_{*} K\right)
$$


For the proof we need two lemmas concerning the fundamental chain complex $W\left(H_{*} K\right)$.

LEMMA 6.3. If $I \in C A$ is cofree, then

$$
\begin{aligned}
& H^{s, t}(W(I))=0, \quad s>0, \\
& H^{0, t}(W(I)) \approx \operatorname{Hom}_{\mathscr{C} A}\left(H_{*} S^{t}, I\right) .
\end{aligned}
$$

Proof. It is easy to show that $I \approx U_{*}(M)$ where $M \in \mathscr{M} A$ is injective. Hence for $s>0$

$$
H^{s, t}(W(I)) \approx \operatorname{Ext}_{\mathscr{M}_{A}}^{s, t}\left(Z_{2}, M\right)=0
$$

and the other part is also easy.

For $n \geqq 0$ consider the functor (2.5)

$$
W^{n}: C A \rightarrow \text { (graded } Z_{2} \text {-modules). }
$$

LEMMA 6.4. If $C \in C A$ is nice as a coalgebra, then

$$
R^{0} W^{n}(C) \approx W^{n}(C), \quad R^{s} W^{n}(C)=0 \text { for } s>0 .
$$

Proof. It suffices to prove the lemma with $\Psi$ in place of $W^{n}$. There is an exact sequence of functors on $\mathscr{C} A$

$$
0 \longrightarrow \Psi \longrightarrow L_{2} \stackrel{\delta^{\Delta}}{\longrightarrow} L_{3}
$$

as in 2.4. Since $R^{0}$ is left exact and $R^{0} L_{r}=L_{r}$ it follows that $R^{0} \Psi=\Psi$. There is a natural exact sequence

$$
0 \longrightarrow P C \longrightarrow \tilde{C} \stackrel{\Delta}{\longrightarrow} \Psi(C)
$$

such that $\Delta$ is onto when $C$ is cofree. This gives a long exact sequence of derived functors containing isomorphisms

$$
R^{s \Psi}(C) \approx R^{s+1} P(C), \quad s \geqq 1 .
$$

If $C$ is nice as a coalgebra, then [B] implies $R^{n} P(C)=0$ for $n \geqq 2$, which proves the lemma.

6.5. Proof of 6.2. Let $(X, e)$ be a cosimplicial resolution of $H_{*} K$. Form a double chain complex $D X$, where for $m, n \geqq 0$

$$
(D X)^{m, n}=W^{m}\left(X^{n}\right)
$$

and consider the two spectral sequences converging to the total cohomology $H^{*}(D X)$. One of them, together with 6.3, shows

$$
\operatorname{Ext}_{\dot{E} A}^{s, *}\left(Z_{2}, H_{*} K\right) \approx H^{s}(D X)
$$

and the other one, together with 6.4 , shows

$$
H^{s}\left(W\left(H_{*} K\right)\right) \approx H^{s}(D X) .
$$

This completes the proof. 
PART II. THE mod 2-LOWER CENTRAL SERIES SPECTRAL SEQUENCE

In this part we prove Theorem 2.6 by making a detailed study of a closely related spectral sequence (\$7) constructed by Rector [R].

7. The mod-2 RLCSSS. The filtration of $G K$ by the $\Gamma_{s} G K$ gives rise to the mod-2 restricted lower central series spectral sequence (abbr. mod-2 RLCSSS), $\left\{\hat{E}^{r} K\right\}$, indexed by

$$
E_{s, q}^{1}(K)=\pi_{q}\left(\Gamma_{s} G K / \Gamma_{s+1} G K\right), \quad d^{r}: \hat{E}_{s, q}^{r}(K) \rightarrow E_{s+r, q-1}^{r}(K) .
$$

Note the change of indexing: the filtration $s$ refers to $\Gamma_{s}$, and the $q$ refers to dimension in $G K$.

It was shown in [R] that for $K$ simply connected and of finite type, $\left\{\hat{E}^{r} K\right\}$ converges to $\pi_{*} K$ modulo odd torsion. The convergence is much slower than for the $\left\{E_{r} K\right\}$ of $\S 2$. Furthermore

$$
\pi_{q}\left(G K / \Gamma_{2} G K\right) \approx \tilde{H}_{q+1} K, \quad \hat{E}_{s, q}^{1}(K) \approx \pi_{q} L_{s}\left(G K / \Gamma_{2} G K\right)
$$

where $L=\bigoplus_{s=1}^{\infty} L_{s}$ is the free restricted Lie algebra functor (see 7.2.).

It follows that $\hat{E}^{1}(K)$ depends functorally on the graded $Z_{2}$-module $\tilde{H}_{*} K$, and we shall describe $\hat{E}^{1}(K)$ in 7.3.

DEFINITION 7.1. A graded restricted Lie algebra (over $Z_{2}$ ) consists of a graded $Z_{2}$-module $B$ with $B_{m}=0$ for $m<0$ together with homomorphisms

$$
[\cdot, \cdot]: B_{m} \otimes B_{n} \rightarrow B_{m+n}, \quad m, n \geqq 0,
$$

and functions

$$
(\cdot)^{[2]}: B_{m} \rightarrow B_{2 m}, \quad m \geqq 0,
$$

such that for $w, x \in B_{m}, y \in B_{n}$, and $z \in B_{q}$ :

(i) $[x, y]=[y, x]$,

(ii) $[x, x]=0$,

(iii) $[[x, y], z]+[[y, z], x]+[[z, x], y]=0$,

(iv) $\left[x, y^{[2]}\right]=[[x, y], y]$,

(v) $(w+x)^{[2]}=w^{[2]}+[w, x]+x^{[2]}$.

$B$ is called a restricted Lie algebra if $B_{m}=0$ for $m \neq 0$.

EXAMPLE. If $\dot{A}$ is a graded associative algebra over $Z_{2}$, define for $x \in A_{m}$ and $y \in A_{n},[x, y]=x y+y x, x^{[2]}=x^{2}$. Then $A$ is a graded restricted Lie algebra.

7.2. Free Lie algebra functors. Let $M$ be a graded $Z_{2}$-module with $M_{n}=0$ for $n<0$, and let

$$
T(M)=\bigoplus_{r=0}^{\infty} T^{r}(M)
$$

be the graded tensor algebra generated by $M$. Then

$$
L(M)=\bigoplus_{r=1}^{\infty} L_{r}(M)
$$


is the graded restricted Lie subalgebra of $T(M)$ generated by $M=T^{1}(M)$. Any homomorphism of $M=L_{1}(M)$ to a graded restricted Lie algebra $B$ extends uniquely to a map $L(M) \rightarrow B$ of graded Lie algebras.

NotATION. Let $\bar{H}_{*} K$ denote $\tilde{H}_{*} K$ with gradation lowered by one.

THEOREM 7.3. If $K$ is a reduced simplicial set, there is a natural isomorphism

$$
L\left(\bar{H}_{*} K\right) \hat{\otimes} \Lambda \stackrel{\approx}{\longrightarrow} E^{1} K
$$

under which

$$
\left(L_{r}\left(\bar{H}_{*} K\right) \hat{\otimes} \Lambda^{s}\right)_{q} \rightarrow E_{2}^{1} s_{r, q}(K) .
$$

This will follow from 8.5 .

8. Simplicial restricted Lie algebras. In order to prove 7.3, we study the natural operations on $\pi_{*} R$ where $R$ is any simplicial restricted Lie algebra over $Z_{2}$.

Definition 8.1. For $x \in \pi_{m} R$ and $y \in \pi_{n} R$ the Lie product $[x, y] \in \pi_{m+n} R$ is the image of $x \otimes y$ under the composition

$$
\pi_{m} R \otimes \pi_{n} R \stackrel{g}{\longrightarrow} \pi_{m+n}(R \otimes R) \stackrel{\mu_{*}}{\longrightarrow} \pi_{m+n} R
$$

where $g$ is the Eilenberg-Zilber map [Mac, p. 239] and $\mu$ is the product in $R$.

Definition 8.2. For $x \in \pi_{m} R$ and $\alpha \in \pi_{n} L K\left(Z_{2}, m\right)$ the composition $x \alpha \in \pi_{n} R$ is the image of $\alpha$ under the map

$$
\bar{f}_{*}: \pi_{n} L K\left(Z_{2}, m\right) \rightarrow \pi_{n} R
$$

where $\bar{f}$ is the Lie extension of a simplicial homomorphism $f: K\left(Z_{2}, m\right) \rightarrow R$ with $f_{*}\left(i_{m}\right)=x$.

For $0 \leqq i \leqq n$ let $\lambda_{i} \in \pi_{n+i} L^{2} K\left(Z_{2}, n\right) \approx Z_{2}$ denote the nonzero element (see [6A]). If $x \in R_{m}$ and $0 \leqq i \leqq m$ then $x \lambda_{i} \in \pi_{m+i} R$, and we define $x^{[2]} \in \pi_{2 m} R$ by $x^{[2]}=x \lambda_{m}$.

Proposition 8.3. The operations [, ] and $(\cdot)^{[2]}$ make $\pi_{*} R$ a graded restricted Lie algebra (over $\left.Z_{2}\right)$. Furthermore

(i) For $x, y \in \pi_{m} R$ and $i<m$

$$
(x+y) \lambda_{i}=x \lambda_{i}+y \lambda_{i} .
$$

(ii) For $x \in \pi_{m} R, y \in \pi_{n} R$, and $i<n$

$$
\left[x, y \lambda_{t}\right]=0 .
$$

(iii) For $x \in \pi_{m} R, 0 \leqq i, k$, and $i+k+1 \leqq m$

$$
(x) \lambda_{i} \lambda_{2 i+1+k}=\sum_{j \geqq 0}\left(\begin{array}{c}
k-1-j \\
j
\end{array}\right)(x) \lambda_{i+k-j} \lambda_{2 i+1+j} .
$$

Proof. Part (iii) is a consequence of [6A]. The others are straightforward using simplicial formulas, of [C2, p. 84], or by universal examples; e.g. for (ii), take $R=L\left(K\left(Z_{2}, m\right) \oplus K\left(Z_{2}, n\right)\right)$. These examples are analyzed by means of 8.4. 
8.4. Decomposition of $L(M \oplus N)$. If $M$ and $N$ are (simplicial) $Z_{2}$-modules, there is an isomorphism [Sch]

$$
D_{1} \oplus D_{2}: L(M) \oplus L(N \otimes T(M)) \rightarrow L(M \oplus N)
$$

where $D_{1}$ and $D_{2}$ are the restricted Lie maps induced respectively by

$$
M \stackrel{\subset}{\longrightarrow} L(M \oplus N)
$$

and by the homomorphisms

$$
N \otimes T^{s}(M) \rightarrow L(M \oplus N)
$$

sending

$$
n \otimes m_{1} \otimes \cdots \otimes m_{s} \rightarrow\left[\cdots\left[n, m_{1}\right], \ldots, m_{s}\right] .
$$

The isomorphism may be iterated to yield a decomposition

$$
\begin{gathered}
L(M \oplus N) \approx L(M) \oplus L(N) \oplus L(N \otimes M) \oplus L(N \otimes M \otimes M) \\
\oplus L(N \otimes M \otimes N) \oplus \cdots .
\end{gathered}
$$

THEOREM 8.5. If $X$ is a simplicial $Z_{2}$-module there is a natural isomorphism

$$
L\left(\pi_{*} X\right) \hat{\otimes} \Lambda \stackrel{\approx}{\longrightarrow} \pi_{*} L X .
$$

Proof. The inclusion

$$
\pi_{*} X=\pi_{*} L_{1} X \subset \pi_{*} L X
$$

extends uniquely to a map $L\left(\pi_{*} X\right) \rightarrow \pi_{*} L X$ of graded restricted Lie algebras. This extends uniquely to a homomorphism

$$
L\left(\pi_{*} X\right) \hat{\otimes} \Lambda \rightarrow \pi_{*} L X
$$

by interpreting the elements $\alpha \in \Lambda$ as composition operations. (The relevant operations are additive by 8.3(i).) This homomorphism is an isomorphism in the case $X=K\left(Z_{2}, n\right)$ by [6A], and thus in the general case by 8.4 .

REMARK 8.6. The operations [, ], ( $)^{[2]}$, and ( $) \lambda_{i}$ on $\pi_{*} L X$ are completely determined by 8.3 and 8.5 . Clearly 8.5 implies 7.3 . It also provides a classification of homotopy operations for simplicial restricted Lie algebras.

9. Samelson products. If $G$ is a simplicial group, the commutator pairing

$$
\langle\cdot, \cdot\rangle: G \wedge G \rightarrow G
$$

with $\langle g, h\rangle=g h g^{-1} h^{-1}$ induces the Samelson product

$$
\langle,\rangle: \pi_{m} G \wedge \pi_{n} G \rightarrow \pi_{m+n} G
$$

for $m, n \geqq 0$, which is bilinear for $m, n \geqq 1$. See for example [C2, p. 103] for a representative simplicial formula. This product in $\pi_{*} G K$ corresponds (with appropriate signs) to the Whitehead product in $\pi_{*} K$. 
THEOREM 9.1. If $K$ is a reduced simplicial set, there are natural pairings

$$
[,]: \hat{E}_{s, t}^{r}(K) \otimes \hat{E}_{u, v}^{r}(K) \rightarrow \hat{E}_{s+u, t+v}^{r}(K)
$$

making $\hat{E}^{r}(K)$ a graded Lie algebra for $r \geqq 1$ and satisfying:

(i) If $r=1$ then [, ] is the Lie product of $\S 8$.

(ii) If $r \geqq 1$, and $x, y \in \hat{E}^{r}(K)$, then

$$
d^{r}[x, y]=\left[d^{r} x, y\right]+\left[x, d^{r} y\right] .
$$

(iii) The product [, ] in $\hat{E}^{r+1}(K)$ is induced by that in $E^{r}(K)$.

(iv) If $K$ is simply connected and of finite type, then the product $[$,$] in \hat{E}^{\infty}(K)$ is induced by the Samelson product in $\pi_{*} G K$.

This theorem is an easy corollary of 9.4 (iii).

REMARK 9.2. It follows by $\S 8$ that in $\hat{E}^{1} K \approx L\left(H_{*} K\right) \hat{\otimes} \Lambda$ the only nontrivial Lie products are those involving elements in the summand $L\left(\bar{H}_{*} K\right)$.

9.3. Generalized Samelson products. The commutator map for a simplicial group $G$ gives a pairing

$$
\langle,\rangle:\left(\Gamma_{r} G / \Gamma_{r+k} G\right) \wedge\left(\Gamma_{s} G / \Gamma_{s+k} G\right) \rightarrow \Gamma_{r+s} G / \Gamma_{r+s+k} G
$$

for $r, s \geqq 1$ and $1 \leqq k \leqq \infty$ where $\Gamma_{\infty} G=*$. This pairing induces the generalized Samelson product $\langle\rangle:, \pi_{m}\left(\Gamma_{r} G / \Gamma_{r+k} G\right) \wedge \pi_{n}\left(\Gamma_{s} G / \Gamma_{s+k} G\right) \rightarrow \pi_{m+n}\left(\Gamma_{r+s} G / \Gamma_{r+s+k} G\right)$ for $m, n \geqq 0$ which is bilinear for $m, n \geqq 1$.

For $k$ fixed,

$$
\left\{\pi_{*}\left(\Gamma_{r} G / \Gamma_{r+k} G\right)\right\}_{r \geqq 1}
$$

has the product $\langle$,$\rangle , and (if k<\infty$ ) has differential

$$
\partial: \pi_{*}\left(\Gamma_{r} G / \Gamma_{r+k} G\right) \rightarrow \pi_{*-1}\left(\Gamma_{r+k} G / \Gamma_{r+2 k} G\right)
$$

given by the boundary map for the fibration

$$
\Gamma_{r+k} G / \Gamma_{r+2 k} G \rightarrow \Gamma_{r} G / \Gamma_{r+2 k} G \rightarrow \Gamma_{r} G / \Gamma_{r+k} G .
$$

Proposition 9.4. If $1 \leqq k \leqq \infty, x \in \pi_{m}\left(\Gamma_{r} G / \Gamma_{r+k} G\right), y \in \pi_{n}\left(\Gamma_{s} G / \Gamma_{s+k} G\right)$, and $z \in \pi_{q}\left(\Gamma_{t} G / \Gamma_{t+k} G\right)$, then:

(i) For $m, n \geqq 1$

$$
\langle x, y\rangle+(-1)^{m n}\langle y, x\rangle=0 .
$$

(ii) For $m, n, q \geqq 1$

$$
(-1)^{m q}\langle\langle x, y\rangle, z\rangle+(-1)^{n m}\langle\langle y, z\rangle, x\rangle+(-1)^{q n}\langle\langle z, x\rangle, y\rangle=0 .
$$

(iii) For $k<\infty$ and $m+n \geqq 1$

$$
\partial\langle x, y\rangle=\langle\partial x, y\rangle+(-1)^{m}\langle x, \partial y\rangle .
$$

Proof. Part (i) follows from the usual properties of the smash pairing. For part (ii), use the expression 
$\langle\langle a, b\rangle, c\rangle\left\langle\langle a, b\rangle,\left\langle c^{-1}, b\right\rangle\right\rangle^{c}\langle\langle b, c\rangle, a\rangle$

$$
\cdot\left\langle\langle b, c\rangle,\left\langle a^{-1}, c\right\rangle\right\rangle^{b}\langle\langle c, a\rangle, b\rangle\left\langle\langle c, a\rangle,\left\langle b^{-1}, a\right\rangle\right\rangle^{b}
$$

which reduces to the identity in any group. For part (iii), consider the pairs

$$
\left(A_{i}, A_{i}^{\prime}\right)=\left(\Gamma_{i} G / \Gamma_{i+2 k} G, \Gamma_{i+k} G / \Gamma_{i+2 k} G\right)
$$

for $i=r, s$ and $r+s$. The commutator pairing $A_{r} \wedge A_{s} \rightarrow A_{r+s}$ produces a diagram:

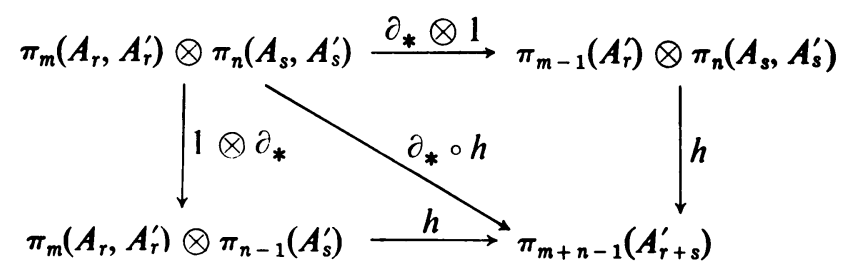

in which $h$ is the smash pairing and

$$
\partial_{*} \circ h=h \circ\left(\partial_{*} \otimes 1\right)+(-1)^{m} h \circ\left(1 \otimes \partial_{*}\right) .
$$

The result follows easily.

10. The cobar spectral sequence. Let $K$ be a reduced simplicial set. When $K$ is simply connected, the cobar construction of Adams [A2] (or the more recent construction of Eilenberg-Moore $[\mathrm{E}, \mathrm{M}]$ ) provides a spectral sequence converging to $H_{*} G K$. We shall construct a version, $\bar{E}(K)$, of this spectral sequence together with a map $\theta: \hat{E}(K) \rightarrow \bar{E}(K)$ corresponding to the Hurewicz map $\pi_{*} G K \rightarrow H_{*} G K$.

For any group $G$, filter the group ring $Z_{2} G$ by powers of the augmentation ideal

$$
Z_{2} G=I^{0} \supset I^{1} \supset \ldots \supset I^{n} \supset I^{n+1} \supset \cdots .
$$

Then $\bigoplus_{n \geqq 0}\left(I^{n} / I^{n+1}\right)$ becomes a Hopf algebra.

LEMMA 10.1. There is a natural homomorphism of Hopf algebras

$$
h: T\left(G / \Gamma_{2} G\right) \rightarrow \bigoplus_{n \geqq 0}\left(I^{n} / I^{n+1}\right)
$$

such that $h$ is an isomorphism if $G$ is a free group.

Proof. Let $\theta: G \rightarrow I$ be the function $\theta(g)=g-e$. Then $\theta$ induces an isomorphism

$$
\theta: G / \Gamma_{2} G \stackrel{\approx}{\longrightarrow} I / I^{2}
$$

and we define $h$ as the unique extension of $\theta$ to the tensor algebra. When $G$ is free, $h$ is an isomorphism by the remarks in [Mac, p. 122].

Let $\left\{\bar{E}^{r}(K)\right\}$ be the spectral sequence arising from the above filtration of $Z_{2} G K$.

$$
\bar{E}_{s, t}^{1}(K)=\pi_{t}\left(I^{s} / I^{s+1}\right), \quad d^{r}: \bar{E}_{s, t}^{r}(K) \rightarrow \bar{E}_{s+r, t-1}^{r}(K) .
$$


THEOREM 10.2. If $K$ is a reduced simplicial set then:

(i) For $r \geqq 1 \bar{E}^{r}(K)$ is a differential graded Hopf algebra with the structure of $\bar{E}^{r+1}(K)$ induced from that of $\bar{E}^{r}(K)$.

(ii) $\bar{E}^{1}(K)$ is the tensor algebra generated by

$$
\bar{E}_{1, *}^{1}(K) \approx \bar{H}_{*} K
$$

with differential $d^{1}$ on $\bar{E}_{1, *}^{1}(K)$ given by the comultiplication

$$
\Delta: \bar{H}_{*} K \rightarrow \bar{H}_{*} K \otimes \bar{H}_{*} K \text {. }
$$

(iii) If $K$ is simply connected then $\left\{\bar{E}^{r}(K)\right\}$ converges to $H_{*} G K$.

Thus $\bar{E}^{\infty}(K)$ is the graded Hopf algebra associated with a decreasing filtration of $H_{*} G K$.

Proof. The convergence part follows from $[\mathrm{D}, \S 11]$ when $K$ is a simply connected suspension, and then follows in general by [C, 4.1]. The structure of $\bar{E}^{\mathrm{I}}(K)$ follows from 10.1. Since the components $H_{m+n} K \rightarrow H_{m} K \otimes H_{n} K$ of $d^{1}$ are natural homomorphisms, they must be given by $\Delta$ if they are nonzero. Using the examples $K=S^{m} \times S^{n}$, one shows they are in fact nonzero. The rest is clear.

REMARK 10.3. It follows immediately from 10.2 (ii) that

$$
\bar{E}_{s, t}^{2}(K) \approx \operatorname{Cotor}_{-s, t+s}^{H, K}\left(Z_{2}, Z_{2}\right)
$$

(see [M,S, II]).

LEMMA 10.4. For each $n \geqq 1, \theta\left(\Gamma_{n} G\right) \subset I^{n}$ and $\theta$ gives a homomorphism

$$
\theta: \Gamma_{n} G / \Gamma_{n+1} G \rightarrow I^{n} / I^{n+1} .
$$

If $G$ is free then the induced map

$$
\theta: L\left(G / \Gamma_{2} G\right) \rightarrow T\left(G / \Gamma_{2} G\right)
$$

is the canonical inclusion.

The proof is straightforward.

Proposition 10.5. The function $\theta: G K \rightarrow Z_{2} G K$ induces a spectral sequence map $\theta: \hat{E}(K) \rightarrow \bar{E}(K)$ such that:

(i) For $r \geqq 1, \theta^{r}: \hat{E}^{r}(K) \rightarrow \bar{E}^{r}(K)$ is a graded Lie algebra map.

(ii) If $K$ is simply connected of finite type then $\theta^{\infty}: \hat{E}^{\infty}(K) \rightarrow \bar{E}^{\infty}(K)$ is induced by the Hurewicz map $\pi_{*} G K \rightarrow H_{*} G K$.

(iii) The map $\theta^{1}: L\left(\bar{H}_{*} K\right) \hat{\otimes} \Lambda \rightarrow T\left(\bar{H}_{*} K\right)$ restricts to the canonical inclusion on the summand $L\left(\bar{H}_{*} K\right)$ and is zero on the other summands.

The proof is straightforward.

11. The chain complex $L\left(\bar{H}_{*} K\right)$. The image complex of the map (10.5)

$$
\theta^{1}: E^{1}(K) \rightarrow \bar{E}^{1}(K)
$$


is the graded restricted Lie algebra $L\left(\bar{H}_{*} K\right)$ with differential

$$
\delta^{\Delta}: L_{s}\left(\bar{H}_{*} K\right) \rightarrow L_{s+1}\left(\bar{H}_{*} K\right), \quad s \geqq 1
$$

determined by

(i) $\delta^{\Delta}[x, y]=\left[\delta^{\Delta} x, y\right]+\left[x, \delta^{\Delta} y\right]$,

(ii) $\delta^{\Delta}\left(x^{[2]}\right)=\left[\delta^{\Delta} x, x\right]$

(iii) for $s=1$

$$
\delta^{\Delta}: \bar{H}_{*} K \rightarrow L_{2}\left(\bar{H}_{*} K\right) \subset \bar{H}_{*} K \otimes \bar{H}_{*} K
$$

is induced by the comultiplication $\Delta$ of $H_{*} K$.

More generally, let $C$ be any homology coalgebra over $Z_{2}$, and define a differential graded restricted Lie algebra $L(\bar{C})$ as above. For $s \geqq 1$ consider

$$
B_{s} L(\bar{C}) \subset \hat{B}_{s} L(\bar{C}) \subset Z_{s} L(\bar{C}) \subset L_{s}(\bar{C})
$$

where $B_{s} L(\bar{C})$ (resp. $Z_{s} L(\bar{C})$ ) are the boundaries (resp. cycles) in $L_{s}(\bar{C}), \hat{B}_{s} L(\bar{C})$ is generated by all $b^{\left[2^{j]}\right]}$ with $b \in B_{r} L(\bar{C}), 2^{j} r=s$. Define homology groups

$$
H_{s} L(\bar{C})=Z_{s} L(\bar{C}) / B_{s} L(\bar{C}), \quad \hat{H}_{s} L(\bar{C})=Z_{s} L(\bar{C}) / \hat{B}_{s} L(\bar{C}) .
$$

Note that $\hat{H}_{*} L(\bar{C})$ is a graded restricted Lie algebra.

The canonical inclusion $L(\bar{C}) \rightarrow T(\bar{C})$ induces a map

$$
i: \hat{H}_{*} L(\bar{C}) \rightarrow P H_{*} T(\bar{C}) \approx P \operatorname{Cotor}^{C}\left(Z_{2}, Z_{2}\right)
$$

where $P$ denotes the primitive element functor.

THEOREM 11.1. The map $i$ is an isomorphism of graded restricted Lie algebras.

Proof. For $s \geqq 2$, we first show that the map

$$
\xi: \hat{B}_{s} L(\bar{C}) \rightarrow \hat{B}_{2 s} L(\bar{C}) / B_{2 s} L(\bar{C})
$$

defined by $\xi(x)=x^{[2]}$ is an isomorphism. Clearly $\xi$ is a homomorphism since $[x, y] \in B_{2 s} L(\bar{C})$ for $x, y \in \hat{B}_{s} L(\bar{C})$. An inverse to $\xi$ is induced by the natural homomorphism $\eta: L_{2 s}(\bar{C}) \rightarrow L_{s}(\bar{C})$ where $\eta[x, y]=0, \eta\left(x^{[2]}\right)=x$.

Choose a set of homogeneous elements $\left\{z_{i}\right\}_{i \in I}$ in $Z_{*} L(\bar{C})$ corresponding to a basis for $\hat{H}_{*} L(\bar{C})$; and choose a set of homogeneous elements $\left\{x_{j}\right\}_{j \in J}$ in $L(\bar{C})$ such that $\left\{\delta^{\Delta} x_{j}\right\}_{j \in J}$ is a basis for $B_{*} L(\bar{C})$. Using the isomorphism $\xi$ it can be seen that

$$
\left\{z_{i}\right\}_{i \in I} \cup\left\{x_{j}\right\}_{j \in J} \cup\left\{\left(\delta^{\Delta} x_{j}\right)^{\left[2^{k}\right]}\right\}_{j \in J, k \geqq 0}
$$

is a $Z_{2}$-basis for $L(\bar{C})$. For each $i \in I$ let $K(i) \subset T(\bar{C})$ be the subcomplex with $Z_{2^{-}}$ basis $\left\{1, z_{i}\right\}$; and for each $j \in J$ let $K(j) \subset T(\bar{C})$ be the subcomplex with $Z_{2}$-basis

$$
\left\{1, x_{j}\right\} \cup\left\{\left(\delta^{\Delta} x_{j}\right)^{n}\right\}_{n \geqq 1} \cup\left\{x_{j}\left(\delta^{\Delta} x_{j}\right)^{n}\right\}_{n \geqq 1} .
$$

Choose a simple ordering of the index sets $I$ and $J$. Define a chain map

$$
h:\left(\bigotimes_{i \in I} K(i)\right) \otimes\left(\bigotimes_{j \in J} K(j)\right) \rightarrow T(\bar{C})
$$

by $h\left(u_{1} \otimes \cdots \otimes u_{r}\right)=u_{1} \cdots u_{r}$. 
The Poincaré-Birkhoff-Witt theorem [M,M, $\S 6]$, shows that $h$ is an isomorphism. Since the complexes $K(j)$ are acyclic, it follows that a $Z_{2}$-basis for $H_{*} T(\bar{C})$ is given by the elements

$$
\left\{z_{i_{1}} \cdots z_{i_{r}} \mid i_{1}<\cdots<i_{r} \in I\right\} .
$$

The theorem now follows from [M,M, §6].

REMARK 11.2. The theorem and its proof show how $H_{*} L(\bar{C})$ may be computed from $P \operatorname{Cotor}^{C}\left(Z_{2}, Z_{2}\right)$ and $B_{*} L(\bar{C})$.

12. Differentials in $\hat{E}(K)$. Let $K$ be a reduced simplicial set. Recall from $\S 8$ that $\Lambda$ operates (on the right) on $\hat{E}^{1}(K)$ and that

$$
\hat{E}^{1}(K) \approx L\left(\bar{H}_{*} K\right) \hat{\otimes} \Lambda \text {. }
$$

THEOREM 12.1. The differentials of $\hat{E}(K)$ satisfy:

(i) If $x \otimes \lambda_{I} \in \bar{H}_{*} K \hat{\otimes} \Lambda^{s}$ with $s \geqq 0$ then

$$
\begin{aligned}
d^{r}\left(x \otimes \lambda_{I}\right) & =0 \text { for } 1 \leqq r<2^{s}, \\
d^{2 s}\left(x \otimes \lambda_{I}\right) & =x\left(\partial \lambda_{I}\right)+\left(\delta^{\Delta} x\right) \lambda_{I}+\sum_{i=1}^{m}\left(x S q^{i}\right) \lambda_{i-1} \lambda_{I}
\end{aligned}
$$

where $m=[\operatorname{deg} x / 2]$.

(ii) If $x \otimes \lambda_{I} \in L_{k}\left(\bar{H}_{*} K\right) \otimes \Lambda^{s}$ with $k>1$ and $s \geqq 0$, then

$$
\begin{aligned}
& d^{r}\left(x \otimes \lambda_{I}\right)=0 \text { for } 1 \leqq r<2^{s}, \\
& d^{2^{s}}\left(x \otimes \lambda_{I}\right)=\left(\delta^{\Delta} x\right) \lambda_{I} .
\end{aligned}
$$

REMARK 12.2. This theorem reduces the computation of $\hat{E}^{2}(K)$ to finding $\hat{E}_{1, *}^{2}(K), \hat{E}_{2, *}^{2}(K)$, and $H_{*} L\left(\bar{H}_{*} K\right)$ (see $\left.\S 11\right)$.

REMARK 12.3. SUSPENSION. Let $S K$ denote the suspension of $K$ as in [K, p. 311]. The canonical function $K \rightarrow S K$, of degree +1 , induces a spectral sequence map

$$
\sigma: \hat{E}(K) \rightarrow \hat{E}(S K)
$$

sending $\hat{E}_{s, t}^{r}(K)$ to $\hat{E}_{s, t+1}^{r}(S K)$ and commuting with the differentials. It is straightforward to show for $r=1$ that

$$
\sigma: L\left(\bar{H}_{*} K\right) \hat{\otimes} \Lambda \rightarrow L\left(\bar{H}_{*} S K\right) \hat{\otimes} \Lambda
$$

is given as follows. For $j, m \geqq 0$ let $e$ denote the composition

$$
\left(L_{2^{j}}\left(\bar{H}_{*} K\right)\right)_{2^{y} m} \stackrel{Q}{\longrightarrow}\left(\bar{H}_{*} K\right)_{m} \stackrel{\text { susp }}{\longrightarrow}\left(\bar{H}_{*} S K\right)_{m+1}
$$

where $Q=$ ident for $j=0$ and $Q=(\eta)^{j}$ for $j>0$ with $\eta$ as in proof of 11.1. If

$$
x \otimes \lambda_{I} \in\left(L_{k}\left(\bar{H}_{*} K\right)\right)_{n} \hat{\otimes} \Lambda^{s}
$$

then

$$
\begin{aligned}
\sigma\left(x \otimes \lambda_{I}\right) & =(e x) \otimes \lambda_{m} \lambda_{2 m} \cdots \lambda_{2^{j-1} m} \lambda_{I} & & \text { for } k=2^{j}, n=2^{j} m \\
& =0 & & \text { otherwise. }
\end{aligned}
$$


Thus $\sigma$ maps $L\left(\bar{H}_{*} K\right) \hat{\otimes} \Lambda$ onto $\bar{H}_{*} S K \hat{\otimes} \Lambda$ and maps $H_{*} K \hat{\otimes} \Lambda$ injectively.

LEMMA 12.4. The differential $d^{1}$ of $E^{1}(K)$ satisfies:

(i) For $x \in \bar{H}_{*} K$

$$
d^{1}(x)=\delta^{\Delta} x+\sum_{i=1}^{m} x S q^{i} \otimes \lambda_{1-1}
$$

where $m=[(\operatorname{deg} x) / 2]$.

(ii) For $x \in L_{k}\left(\bar{H}_{*} K\right)$ with $k>1$

$$
d^{1}(x)=\delta^{\Delta} x .
$$

Proof. The component $H_{*} K \rightarrow H_{*} K \hat{\otimes} \Lambda^{1}$ of $d^{1}$ is determined from [6A] using the iterated suspension map $\sigma$.

The components

$$
L_{k}\left(\bar{H}_{*} K\right) \rightarrow L_{k+1}\left(\bar{H}_{*} K\right), \quad k \geqq 1,
$$

of $d^{1}$ are determined using the spectral sequence Hurewicz map of $\S 10$. The proof of (ii) is completed using the fact that $\hat{E}^{1}(K)$ is a differential Lie algebra (9.1), and $\hat{E}_{s, *}^{1}(K) \approx L_{s}\left(\bar{H}_{*} K\right)$ for $s$ odd.

12.5. Composition. The composition product

$$
\pi_{n}\left(\Gamma_{r} G / \Gamma_{r+1} G\right) \times \pi_{j}\left(\Gamma_{s} F S^{n} / \Gamma_{s+1} F S^{n}\right) \rightarrow \pi_{j}\left(\Gamma_{r s} G / \Gamma_{r s+1} G\right)
$$

is defined as usual when $G$ is a simplicial group and $F$ is the free group functor. Let

$$
\bar{u} \in \pi_{n}\left(\Gamma_{r} G / \Gamma_{r+1} G\right), \quad \bar{v} \in \pi_{j-1}\left(\Gamma_{s} F S^{n-1} / \Gamma_{s+1} F S^{n-1}\right)
$$

denote the respective images of elements

$$
\begin{array}{ll}
u \in \pi_{n}\left(\Gamma_{r} G / \Gamma_{r+h} G\right), & h \geqq 1, \\
v \in \pi_{j-1}\left(\Gamma_{s} F S^{n-1} / \Gamma_{s+k} F S^{n-1}\right), & k \geqq 1,
\end{array}
$$

under the canonical maps. Let

$$
\sigma \bar{v} \in \pi_{j}\left(\Gamma_{s} F S^{n} / \Gamma_{s+1} F S^{n}\right)
$$

be the suspension of $\bar{v}$ (see 12.3). Consider the composition

$$
\bar{u} \circ(\sigma \bar{v}) \in \pi_{j}\left(\Gamma_{r s} G / \Gamma_{r s+1} G\right) .
$$

The following lemma and its proof are similar to [6A, §3].

LEMMA 12.6. There is an element

$$
w \in \pi_{,}\left(\Gamma_{r s} G / \Gamma_{r s+m} G\right)
$$

where $m=\operatorname{Min}(s h, r k)$ such that:

(i) $\bar{u} \circ(\sigma \bar{v})$ is the image of $w$ under the canonical map.

(ii) The element

$$
\partial w \in \pi_{j-1}\left(\Gamma_{r s+m} G / \Gamma_{r s+m+1} G\right)
$$


satisfies

$$
\begin{array}{llrl}
\partial w & =(\partial u) \circ \bar{v} & & \text { if } s h<r k, \\
\partial w & =\bar{u} \circ(\sigma \partial v) & & \text { if } r k<s h, \\
\partial w & =(\partial u) \circ \bar{v}+\bar{u} \circ(\sigma \partial v) & & \text { if } r k=s h .
\end{array}
$$

Proof. Represent $u$ by $\mu \in\left(\Gamma_{r} G\right)_{n}$ such that $d_{n} \mu \in\left(\Gamma_{r+h} G\right)_{n-1}$ and $d_{i} \mu=*$ for $i<n$. Represent $v$ by $\nu \in\left(\Gamma_{s} F S^{n-1}\right)_{j-1}$ such that $d_{0} \nu \in\left(\Gamma_{s+k} F S^{n-1}\right)_{j-2}$ and $d_{i} \nu=*$ for $i>0$. Write $\nu$ in the form $B\left(i^{n-1}\right)$ where $B$ is a formula involving only degeneracy operators and the group operations of product and inverse. Then $B(\mu)$ $\in\left(\Gamma_{r s} G\right)_{j}$ represents $\bar{u} \circ(\sigma \bar{v}) \in \pi_{j}\left(\Gamma_{r s} G / \Gamma_{r s+1} G\right)$ and it is straightforward to show:

$$
\begin{aligned}
d_{i}(B(\mu)) & =C(\mu) & & \text { for } i=0, \\
& =* & & \text { for } 0<i<j, \\
& =B\left(d_{n} \mu\right) & & \text { for } i=j,
\end{aligned}
$$

where $C$ is a formula of the same sort as $B$ with $C\left(i^{n-1}\right)=d_{0} \nu$. Clearly

$$
C(\mu) \in\left(\Gamma_{r(s+k)} G\right)_{j-1}
$$

represents

$$
\bar{u} \circ(\sigma \partial v) \in \pi_{j-1}\left(\Gamma_{r s+r k} G / \Gamma_{r s+r k+1} G\right)
$$

and $B\left(d_{n} \mu\right) \in\left(\Gamma_{(r+h) s} G\right)_{j-1}$ represents

$$
\partial u \circ \bar{v} \in \pi_{j-1}\left(\Gamma_{r s+s h} G / \Gamma_{r s+s h+1} G\right) .
$$

The result now follows easily.

12.7. Proof of 12.1. Let

$$
x \otimes \lambda_{I} \in\left(L_{k}\left(\bar{H}_{*} K\right) \hat{\otimes} \Lambda^{s}\right)_{j}
$$

with $\operatorname{deg} x=n$ and $s>0$. Then

$$
\begin{gathered}
x \in \pi_{n}\left(\Gamma_{k} G K / \Gamma_{k+1} G K\right), \\
\lambda_{I} \in \pi_{j-1}\left(\Gamma_{2^{s}} F S^{n-1} / \Gamma_{2^{s}+1} F S^{n-1}\right), \\
x \otimes \lambda_{I}=x \circ\left(\sigma \lambda_{I}\right) .
\end{gathered}
$$

(We usually denote $\sigma \lambda_{I}$ by $\lambda_{I}$.) By [6A] there exists

$$
v \in \pi_{j-1}\left(\Gamma_{2^{s}} F S^{n-1} / \Gamma_{2^{s+1}} F S^{n-1}\right)
$$

mapping to $\lambda_{I}$ and satisfying

$$
\partial v=\partial \lambda_{I} \in \pi_{j-2}\left(\Gamma_{2^{s+1}} F S^{n-1} / \Gamma_{2^{s+1}+1} F S^{n-1}\right)
$$

where $\partial \lambda_{I}$ is as in 2.3. It follows by 12.6 that $d^{r}\left(x \otimes \lambda_{I}\right)=0$ for $r<2^{s}$; and $d^{2^{s}}\left(x \otimes \lambda_{I}\right)$ is represented by

$$
\begin{array}{ll}
\left(d^{1} x\right) \lambda_{I} \in \pi_{j-1}\left(\Gamma_{2^{s}(k+1)} G K / \Gamma_{2^{s}(k+1)+1} G K\right) & \text { for } k>1, \\
\left(d^{1} x\right) \lambda_{I}+x\left(\partial \lambda_{I}\right) \in \pi_{j-1}\left(\Gamma_{2^{s+1}} G K / \Gamma_{2^{s+1}+1} G K\right) & \text { for } k=1 .
\end{array}
$$

The result now follows from the description of $d^{1} x$ in 12.4. 
13. Proof of Theorem 2.6. Let us use temporarily the auxiliary filtration $\left\{f_{s} G K\right\}_{s \geqq 0}$, where

$$
f_{2 j} G K=\Gamma_{2^{j}} G K, \quad f_{2 j+1} G K=\Gamma_{2^{j}+1} G K .
$$

This gives rise to a spectral sequence $\left\{e^{r}(K)\right\}$, where

$$
e_{s, q}^{1}(K)=\pi_{q}\left(f_{s} G K / f_{s+1} G K\right), \quad d^{r}: e_{s, q}^{r}(K) \rightarrow e_{s+r, q-1}^{r}(K) .
$$

Theorem 2.6 follows easily from:

Proposition 13.1. If $K$ is a reduced simplicial set with $H_{*} K$ nice, then $e_{s, q}^{2}(K)=0$ for $s$ odd, and the chain complex

$$
e_{0, *}^{2}(K) \stackrel{d^{2}}{\longrightarrow} \ldots \longrightarrow e_{2 j, *}^{2}(K) \stackrel{d^{2}}{\longrightarrow} e_{2 j+2, *}^{2}(K) \longrightarrow \ldots
$$

is isomorphic to $W\left(H_{*} K\right)$.

This will be proved in 13.4.

13.2. $L\left(\bar{H}_{*} K\right)$ for $H_{*} K$ nice. It follows from 11.1 and [B] that when $H_{*} K$ is nice:

(i) $\hat{H}_{s} L\left(\bar{H}^{*} K\right)=0$ unless $s=2^{j}, j \geqq 0$.

(ii) The Lie products in $\hat{H}_{*} L\left(\bar{H}_{*} K\right)$ are zero except possibly for those of the form

$$
\text { [ , ]: } \hat{H}_{1} L\left(\bar{H}_{*} K\right) \otimes \hat{H}_{1} L\left(\bar{H}_{*} K\right) \rightarrow \hat{H}_{2} L\left(\bar{H}_{*} K\right) .
$$

(iii) For $j \geqq 1$

$$
()^{[2]}: \hat{H}_{2^{j}} L\left(\bar{H}_{*} K\right) \rightarrow \hat{H}_{2^{j+1}} L\left(\bar{H}_{*} K\right)
$$

is an isomorphism.

Define for $j \geqq 1$

$$
\Psi_{2^{\jmath}}\left(H_{*} K\right)=Z_{2^{\jmath}} L\left(\bar{H}_{*} K\right) / \widetilde{B}_{2^{\jmath}} L\left(\bar{H}_{*} K\right)
$$

where $\widetilde{B}_{2} J\left(\bar{H}_{*} K\right) \subset L_{2^{\prime}}\left(\bar{H}_{*} K\right)$ is generated by all $b^{\left[2^{t}\right]}$ with $b \in B_{2^{k}} L\left(\bar{H}_{*} K\right), k=$ $j-1>1$.

It follows from the above that when $H_{*} K$ is nice:

(i) For $j \geqq 1$

$$
()^{[2]}: \Psi_{2^{j}}\left(H_{*} K\right) \rightarrow \Psi_{2^{j+1}}\left(H_{*} K\right)
$$

is an isomorphism.

(ii) The functor $\Psi_{2}$ coincides with $\Psi$ in 2.4 .

For $j \geqq 1$ consider the spectral sequence $\left\{\mathscr{E}^{r}(K)\right\}$ with

$$
\begin{aligned}
\mathscr{E}_{s, t}^{1}(K) & =\pi_{t}\left(\Gamma_{s} G K / \Gamma_{s+1} G K\right) & & \text { for } 2^{j} \leqq s \leqq 2^{j+1} \\
& =0 & & \text { otherwise }
\end{aligned}
$$

arising from the obvious filtration of $\Gamma_{2}{ }^{3} G K / \Gamma_{2^{j+1}+1} G K$. 
LEMMA 13.3. If $H_{*} K$ is nice then $\mathscr{E}_{s, t}^{2^{j}}(K)=0$ unless $s=2^{j}, 2^{j+1}$. Furthermore

$$
\begin{aligned}
\mathscr{E}_{2^{j}, *}^{2^{j}}(K) & \approx\left(\bar{H}_{*} K \hat{\otimes} \Lambda^{j}\right) \oplus\left(\bigoplus_{i=1}^{j} Z_{2^{i}} L\left(\bar{H}_{*} K\right) \hat{\otimes} \Lambda^{j-i}\right), \\
\mathscr{E}_{2^{j+1}, *}^{2^{j}}(K) & \approx\left(\bar{H}_{*} K \hat{\otimes} \Lambda^{j+1}\right) \oplus\left(\bigoplus_{i=1}^{j+1} L_{2^{i}} \bar{H}_{*} K / \widetilde{B}_{2} L\left(\bar{H}_{*} K\right) \hat{\otimes} \Lambda^{j-i+1}\right) .
\end{aligned}
$$

Proof. The spectral sequence $\mathscr{E}(K)$ may be completely computed in the required range using $12.1,13.2$, the proof of 11.1 , and one other technique. Namely, there is a map $f: K \rightarrow X$ such that $X$ is a product of $K\left(Z_{2}, n\right)$ 's and $f_{*}: H_{*} K \rightarrow H_{*} X$ is a monomorphism. Since $\hat{H}_{s} L\left(\bar{H}_{*} X\right)=0$ for $s>0$, it is possible to show certain differentials vanish in $\mathscr{E}(X)$ and consequently in $\mathscr{E}(K)$ by naturality.

13.4. Proof of 13.1. The above lemma implies $e_{s, *}^{2}(K)=0$ for $s$ odd, $e_{0, *}^{2}(K)$ $\approx \bar{H}_{*} K$ and for $j \geqq 1$

$$
e_{2 j, *}^{2}(K) \approx\left(\bar{H}_{*} K \hat{\otimes} \Lambda^{j}\right) \oplus\left(\bigoplus_{i=1}^{j} \Psi_{2^{i}}\left(H_{*} K\right) \hat{\otimes} \Lambda^{j-i}\right) .
$$

Thus by 13.2

$$
e_{2 j, *}^{2}(K) \approx W^{j}\left(H_{*} K\right)
$$

and it remains only to show that the differential $d^{2}$ of $e^{2}(K)$ corresponds to $\delta$ in $W\left(H_{*} K\right)$. For the components 2.5 (i) of the differential, this follows easily from 12.1 . For the components 2.5 (ii), it suffices by naturality (see proof of 13.3) to suppose $K$ is a product of $K\left(Z_{2}, n\right)$ 's. Then

$$
\Delta: \tilde{H}_{*} K \rightarrow \Psi\left(H_{*} K\right)
$$

is onto and the last components may be deduced from the first.

\section{REFERENCES}

[A1] J. F. Adams, On the structure and applications of the Steenrod algebra, Comment. Math. Helv. 32 (1958), 180-214. MR 20 \#2711.

[A2] - - On the cobar construction, Colloque de topologie algébrique (Louvain, 1956), Georges Thone, Liège, 1957, pp. 81-87. MR 19, 759.

[An] M. André, Méthode simpliciale en algèbre homologique et algèbre commutative, Lecture Notes in Math., no. 32, Springer-Verlag, Berlin, 1967. MR 35 \#5493.

[6A] Bousfield, Curtis, Kan, Quillen, Rector and Schlesinger, The mod-p lower central series and the Adams spectral sequence, Topology 5 (1966), 331-342.

[B] A. K. Bousfield, Nice homology coalgebras, Trans. Amer. Math. Soc. 148 (1970), 473489.

[B,K] A. K. Bousfield and D. M. Kan, The homotopy spectral sequence of a space with coefficients in a ring, (to appear).

[Cl] E. B. Curtis, Some relations between homotopy and homology, Ann. of Math. (2) 82 (1965), 386-413. MR 32 \#1704.

[C2] —- Simplicial homotopy theory, Aarhus Univ. Lecture Notes, Matematisk Institut, Aarhus, Denmark, 1967.

[C3] - Some nonzero homotopy groups of spheres, Bull. Amer. Math. Soc. 75 (1969), 541-544. 
[D] A. Dold, Homology of symmetric products and other functors of complexes, Ann. of Math. (2) 68 (1958), 54-80. MR 20 \#3537.

[E,M] S. Eilenberg and J. C. Moore, Homology and fibrations. I: Coalgebras, cotensor product and its derived functors, Comment. Math. Helv. 40 (1966), 199-266. MR 34 \#3579.

[K] D. M. Kan, A combinatorial definition of homotopy groups, Ann. of Math. (2) 67 (1958), 282-312. MR 22 \#1897.

[K,W] D. M. Kan and G. W. Whitehead, The reduced join of two spectra, Topology 3 (1965), 239-261. MR 31 \#2720.

[Mac] S. MacLane, Homology, Die Grundlehren der math. Wissenschaften, Band 114, Springer-Verlag, Berlin, 1963. MR 28 \#122.

[M,P] W. Massey and F. Peterson, The mod 2 cohomology structure of certain fibre spaces, Mem. Amer. Math. Soc. No. 74 (1967). MR 37 \#2226.

[M,M] J. Milnor and J. C. Moore, On the structure of Hopf algebras, Ann. of Math. (2) 81 (1965), 211-264. MR 30 \#4259.

[M,S] J. C. Moore and L. Smith, Hopf algebras and multiplicative fibrations. II, Amer. J. Math. 90 (1968), 1113-1150. MR 38 \#6599.

[R] D. Rector, An unstable Adams spectral sequence, Topology 5 (1966), 343-346. MR 33 \#8003.

[Sch] J. W. Schlesinger, The semi-simplicial free Lie ring, Trans. Amer. Math. Soc. 122 (1966), 436-442. MR 33 \#8001.

[Sm] L. Smith, Homological algebra and the Eilenberg-Moore spectral sequence, Trans. Amer. Math. Soc. 129 (1967), 58-93. MR 35 \#7337.

[S,E] N. E. Steenrod and D. B. A. Epstein, Cohomology operations, Ann. of Math. Studies, no. 50, Princeton Univ. Press, Princeton, N. J., 1962. MR 26 \#3056.

BRANDEIS UNIVERSITY,

Waltham, Massachusetts 02154

Massachusetts Institute of TeChNology,

CAMBridge, MASSAChuSETTS 02139 